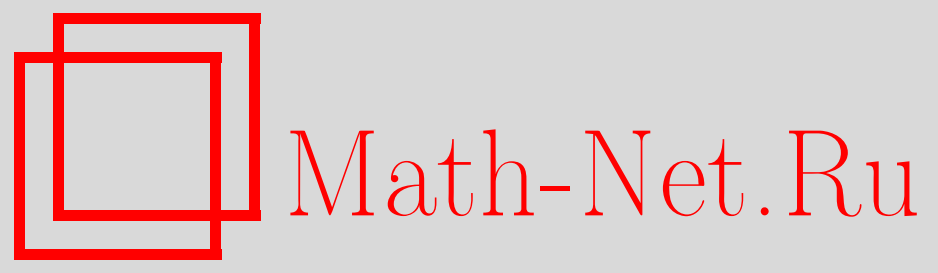

E. B. Dynkin, Exit laws and excessive functions for superprocesses, Теория вероятн. и ее примен., 1999, том 44, выпуск 4, 880-885

DOI: https://doi.org/10.4213/tvp1074

Использование Общероссийского математического портала Math-Net.Ru подразумевает, что вы прочитали и согласны с пользовательским соглашением

http://www . mathnet.ru/rus/agreement

Параметры загрузки:

IP : 3.85 .183 .62

26 апреля 2023 г., 17:02:18

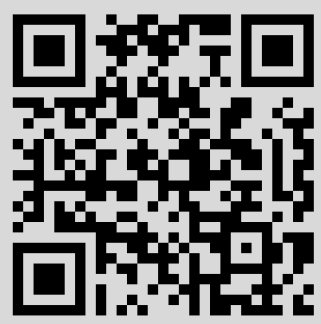


3. Aliev F. A. Characterization of distributions through weak records. - J. Appl. Statist. Sci., 1998, v. 8, № 1, p. 13-16.

4. Kirmani S. N.U.A., Beg M.I. On characterization of distributions by expected records. - Sankhyā, 1984, v. 49, p. 463-465.

5. Nagaraja $H . N$. On the expected values of record values. - Austral. J. Statist., 1978, v. 20, № 2, p. 176-182.

6. Nagaraja $H$. N. Record values and related statistics: A review. - Comm. Statist. Theory Methods, 1988, v. 17, p. 2223-2238.

7. Nevzorov V. B. Records. - Theory Probab. Appl., 1987, v. 32, p. 201-228.

8. Степанов $A . B$. Характеризационная теорема для слабых рекордов. - Теория вероятн. и ее примен., 1993, т. XXXVIII, в. 3, с. 586-590.

9. Vervaat $W$. Limit theorems for records from discrete distributions. - Stochastic Process. Appl., 1973, v. 1, p. 317-334.

Поступила в редакцию 5.V.1998

(c) $1999 \mathrm{r}$.

DYNKIN E. B.*

\section{EXIT LAWS AND EXCESSIVE FUNCTIONS FOR SUPERPROCESSES ${ }^{1)}$}

Пусть $\xi$ есть марковский процесс с переходной функцией $p(r, x ; t, d y)$, a $X$ - соответствуюший суперпроцесс Доусона-Ватанабэ (т.е. суперпроцесс с характеристикой ветвления $\left.\psi(u)=\gamma u^{2}\right)$. Обозначим через $\mathscr{P}$ переходную функцию $X$ и положим

$$
p_{n}(r, x ; t, d y)=\prod_{i=1}^{n} p\left(r, x_{i} ; t, d y_{i}\right) .
$$

Любому закону выхода $\ell$ для $p_{n}$ соответствует закон выхода $L_{\ell}$ для $\mathscr{P}$ такой, что $L_{\ell}^{t}(\mu)$ есть полином степени $n$ по $\mu$ со старшим членом $\left\langle\ell^{t}, \mu^{n}\right\rangle$ для любого $t$. Любой полиномиальный закон выхода для $\mathscr{P}$ допускает единственное представление в виде $L_{\ell_{1}}+\cdots+L_{\ell_{n}}$, где $\ell_{k}$ есть закон выхода для $p_{k}$.

Клюиевые слова и фразы: марковский процесс, суперпроцесс ДоусонаВатанабэ, полиномиальный закон выхода для $\mathscr{P}$.

\section{Dedicated to Yu. V. Prokhorov on his 70th birthday}

\section{Action of the Dawson-Watanabe semigroup on polynomials}

1.1. The Dawson-Watanabe superprocess. With every measurable space $(E, \mathscr{B})$ another measurable space $(\mathscr{M}, \mathscr{B} \mathscr{M})$ is associated: $\mathscr{M}$ is the set of all finite measures on $(E, \mathscr{B})$ and $\mathscr{B} \mathscr{M}$ is the $\sigma$-algebra in $\mathscr{M}$ generated by the functions $F(\mu)=\mu(B)$ with $B \in \mathscr{B}$. To every Markov transition function $p$ in $(E, \mathscr{B})$ there corresponds a Markov transition function $\mathscr{P}$ in $(\mathscr{M}, \mathscr{B} \mathscr{M})$ such that, for every $f \in p \mathscr{B}, 2)$

$$
\int_{\mathscr{M}} \mathscr{P}(r, \mu ; t, d \nu) e^{-\langle f, \nu\rangle}=e^{-\langle u, \nu\rangle},
$$

* Cornell University, Department of Mathematics, White Hall, Ithaca, NY, 14853; e-mail: ebdl@cornell.edu

1) Partially supported by National Science Foundation Grant DMS-9970942.

2) This writing means that $f$ is a $\mathscr{B}$-measurable function from $E$ to $[0, \infty]$. We write $f \in \mathbf{p} \mathscr{B}$ if $f \in p \mathscr{B}$ and $f$ is bounded. 
where

$$
u(r, x)+\gamma \int_{r}^{t} p(r, x ; s, d y) u^{2}(s, y) d s=\int_{E} p(r, x ; t, d y) f(y)
$$

( $\gamma$ is a positive constant). If $p$ is the transition function of a right Markov process $\xi=$ $\left(\xi_{t}, \Pi_{r, x}\right)$, then $\mathscr{P}$ is the transition function of a right Markov process $\left.X=\left(X_{t}, P_{r, \mu}\right){ }_{\cdot}{ }^{3}\right)$ We call $X$ the Dawson-Watanabe superprocess. The Markov semigroups of $\xi$ and $X$ are denoted $T_{t}^{r}$ and $\mathscr{T}_{t}^{r}$.

Both transition functions $p$ and $\mathscr{P}$ are defined on the same time interval $I$. Without any loss of generality we can assume that $I=\mathbb{R}_{+}$.

1.2. Martingale characterization of $X$. We use the following property of the Dawson-Watanabe superprocess: for all $r<u \in \mathbb{R}_{+}, \mu \in \mathscr{M}$ and $\varphi \in \mathbb{p} \mathscr{B}$,

$$
\left\langle T_{u}^{t} \varphi, X_{t}\right\rangle=\int X_{t}(d x) p(t, x ; u, d y) \varphi(y)
$$

is a continuous square integrable martingale on $(r, u)$ relative to $P_{r, \mu}$ with the quadratic variation $\gamma\left\langle\left(T_{u}^{t} \varphi\right)^{2}, X_{t}\right\rangle d t$ (see, e.g., [2]; in a slightly different form this was proved earlier in [5]).

1.3. Polynomials on the space $\mathscr{M}$. Denote by $S^{k}$ the cone of finite positive measurable symmetric functions on the product space $\left(E^{k}, \mathscr{B}^{k}\right)$ of $k$ replicas of $(E, \mathscr{B})$. To every $\mu \in \mathscr{M}$ there corresponds a measure

on $\left(E^{k}, \mathscr{B}^{k}\right)$. We deal with functions on $\mathscr{M}$ of the form

$$
\mu^{k}\left(d x_{1}, \ldots, d x_{k}\right)=\mu\left(d x_{1}\right) \cdots \mu\left(d x_{k}\right)
$$

$$
F(\mu)=\sum_{1}^{n}\left\langle f_{k}, \mu^{k}\right\rangle \quad \text { with } f_{k} \in S^{k} .
$$

We call them polynomials in $\mu$. Note that the coefficients $f_{k}$ are uniquely determined by $F$. Indeed, $k ! f_{k}\left(x_{1}, \ldots, x_{k}\right)$ is the coefficient at $\lambda_{1} \cdots \lambda_{k}$ in the polynomial $F\left(\lambda_{1} \delta_{x_{1}}+\right.$ $\left.\cdots+\lambda_{k} \delta_{x_{k}}\right)$.

Operators $B_{k}: S^{k} \rightarrow S^{k-1}$ are defined by the formula

$$
B_{k} f\left(x_{1}, \ldots, x_{k}\right)=\frac{k \gamma}{2} \sum_{\sigma} f\left(x_{\sigma_{1}}, \ldots, x_{\sigma_{k}}\right),
$$

where $\sigma$ runs over all monotone increasing mappings from $\{1, \ldots, k\}$ onto $\{1, \ldots, k-1\}$. For instance,

Formula

$$
B_{3} f\left(x_{1}, x_{2}\right)=\frac{3 \gamma}{2}\left[f\left(x_{1}, x_{1}, x_{2}\right)+f\left(x_{1}, x_{2}, x_{2}\right)\right] .
$$

$$
p_{k}(r, x ; t, d y)=\prod_{1}^{k} p\left(r, x_{i} ; t, d y_{i}\right)
$$

defines a transition function in $\left(E^{k}, \mathscr{B}^{k}\right)$. The corresponding semigroup $T_{k}(r, t)$ preserves cone $S^{k}$.

1.4. Action of $\mathscr{T}_{t}^{r}$ on polynomials.

Theorem 1.1. For every $r<t$ and every $f \in S^{k}$

$$
P_{r, \mu}\left\langle f, X_{\imath}^{k}\right\rangle=\sum_{j=1}^{k}\left\langle R_{j k}(r, t) f, \mu^{j}\right\rangle,
$$

where

$$
R_{k k}(r, t)=T_{k}(r, t), \quad R_{j k}(r, t)=\int_{r}^{t} R_{j, k-1}(r, s) B_{k} T_{k}(s, t) d s \quad \text { for } j<k .
$$

3) The definition of a right process in the nonhomogeneous setting and the construction of $X$ can be found, e.g., in [3]. 
P r o o f. Formula (1.7) follows by induction in $k$ from the equation

$$
P_{r, \mu}\left\langle f, X_{t}^{k}\right\rangle=\left\langle T_{k}(r, t) f, \mu^{k}\right\rangle+P_{r, \mu} \int_{r}^{t}\left\langle B_{k} T_{k}(s, t) f, X_{s}^{k-1}\right\rangle d s .
$$

To establish (1.9) we note that if $\eta$ and $\tilde{\eta}$ are two measures on $\left(E^{k}, \mathscr{B}^{k}\right)$ and if

$$
\int f d \eta=\int f d \tilde{\eta}
$$

for all $f$ of the form

$$
f(x)=\varphi\left(x_{1}\right) \cdots \varphi\left(x_{k}\right) \quad \text { with } \varphi \in \mathbf{p} \mathscr{B},
$$

then (1.10) holds for all $f \in S^{k}$. Therefore it is sufficient to prove (1.9) for functions of the form (1.11). For these functions

$$
\left\langle f, X_{t}^{k}\right\rangle=\left\langle\varphi, X_{t}\right\rangle^{k}
$$

Fix $t$ and put $h^{s}=T_{t}^{s} \varphi$. Consider the martingale $Y_{s}=\left\langle h^{s}, X_{s}\right\rangle$ on the interval $(r, t)$. As we know, its quadratic variation is equal to $\gamma\left\langle\left(h^{s}\right)^{2}, X_{s}\right\rangle d s$ and, by the Itô formula,

$$
Y_{t}^{k}=Y_{r}^{k}+\int_{r}^{t} k Y_{s}^{k-1} d Y_{s}+\frac{1}{2} k(k-1) \int_{r}^{t} Y_{s}^{k-2} \gamma\left\langle\left(h^{s}\right)^{2}, X_{s}\right\rangle d s .
$$

Therefore,

$$
P_{r, \mu} Y_{t}^{k}=P_{r, \mu} Y_{r}^{k}+\frac{1}{2} \gamma k(k-1) P_{r, \mu} \int_{r}^{t} Y_{s}^{k-2}\left\langle\left(h^{s}\right)^{2}, X_{s}\right\rangle d s
$$

which implies (1.9). Theorem 1.1 is proved.

1.5. Properties of $R_{j k}$. It follows from (1.8) that, for all $j<k$,

$$
R_{j k}(r, t)=\int_{r<s_{j}<s_{j+1}<\cdots<s_{j_{k-1}}<t} T_{j}\left(r, s_{j}\right) B_{j+1} T_{j+1}\left(s_{j}, s_{j+1}\right) \cdots B_{k} T_{k}\left(s_{k-1}, t\right) d s_{j} \cdots d s_{k-1} .
$$

For instance,

$$
R_{j, j+2}(r, t)=\int_{r<s_{j}<s_{j+1}<t} T_{j}\left(r, s_{j}\right) B_{j+1} T_{j+1}\left(s_{j}, s_{j+1}\right) B_{j+2} T_{j+2}\left(s_{j+1}, t\right) d s_{j} d s_{j+1} .
$$

Formula (1.3) implies that, for all $r<s<t, i \leqslant j \leqslant k$,

$$
\sum_{j=i}^{k} R_{i j}(r, s) R_{j k}(s, t)=R_{i k}(r, t) .
$$

(The semigroup property of operators $R_{i i}(r, t)=T_{i}(r, t)$ is a particular case of (1.14) corresponding to $i=j=k$.)

1.6. Time-homogeneous setting. In this setting, it is convenient to assume that $I=\mathbf{R}$. If the transition function $p$ is stationary (that is, if $p(r, x ; t, d y)=p_{t-r}(x, d y)$ ), then the Dawson-Watanabe superprocess has also a stationary transition function $\mathscr{P}_{t}(\mu, d \nu)$. The operator

$$
G_{\lambda}^{k}=\int_{0}^{\infty} e^{-\lambda t} T_{k}(t) d t
$$

is the resolvent of the semigroup $T_{k}(t)=T_{k}(r, t+r)$. We denote resolvent corresponding to $\mathscr{P}$ by $\mathscr{G}_{\lambda}$.

Theorem 1.2. In the time-homogeneous case,

$$
\int_{\mathscr{M}} \mathscr{P}_{t}(\mu, d \nu)\left\langle f, \nu^{k}\right\rangle=\sum_{j=1}^{k}\left\langle R_{j k}(t) f, \mu^{j}\right\rangle
$$

where

$$
R_{k k}(t)=T_{k}(t), \quad R_{j k}(t)=\int_{0}^{t} R_{j, k-1}(s) B_{k} T_{k}(t-s) d s \quad \text { for } j<k .
$$


If

$$
F(\nu)=\left\langle f, \nu^{k}\right\rangle \text { with } f \in S^{k}
$$

then

$$
\mathscr{G}_{\lambda} F(\mu)=\sum_{j=1}^{k}\left\langle G_{\lambda}^{j} B_{j+1} G_{\lambda}^{j+1} \cdots B_{k} G_{\lambda}^{k} f, \mu^{j}\right\rangle .
$$
(1.19).

P r o o f. Formulae (1.16)-(1.17) follow from (1.7)-(1.8). Clearly, they imply (1.18)-

\section{Exit laws}

2.1. Construction of polynomial $\mathscr{P}$-exit laws. Suppose that $p$ is a Markov transition function in a measurable space $(E, \mathscr{B})$. Let $0<\beta \leqslant \infty$ and let a function $\ell^{t} \in p \mathscr{B}$ not identically equal to $\infty$ be given for every $t \in(0, \beta)$. We say that $\ell$ is a $p$-exit law at time $\beta$ and we write $\ell \in \mathscr{L}_{\beta}(p)$ if $T_{t}^{r} \ell^{t}=\ell^{r}$ for all $r<t<\beta$.

Theorem 2.1. To every $\ell \in \mathscr{L}_{\beta}\left(p_{n}\right)$ there corresponds a $\mathscr{P}$-exit law

$$
L_{\ell}^{t}(\mu)=\left\langle\ell^{t}, \mu^{n}\right\rangle+\sum_{j=1}^{n-1} \int_{t}^{\beta}\left\langle R_{j, n-1}(t, u) B_{n} \ell^{u}, \mu^{j}\right\rangle d u .
$$

If $L \in \mathscr{L}_{\beta}(\mathscr{P})$ and if, for every $t, L^{t}$ has the form (1.4), then

$$
L=\sum_{j=1}^{n} L_{\ell_{j}}
$$

where $\ell_{j} \in \mathscr{L}_{\beta}\left(p_{j}\right)$.

P r o of. $1^{\circ}$. By using (1.7), we establish that

$$
L^{t}(\mu)=\sum_{k=1}^{n}\left\langle f_{k}^{t}, \mu^{k}\right\rangle \quad \text { with } f_{k}^{t} \in S^{k}
$$

satisfies the condition

$$
\mathscr{T}_{t}^{r} L^{t}=L^{r}
$$

if and only if

$$
f_{k}^{r}=\sum_{j=k}^{n} R_{k j}(r, t) f_{j}^{t} \quad \text { for } k=1, \ldots, n .
$$

$2^{\circ}$. Function (2.1) has the form (2.3) with

$$
f_{n}^{t}=\ell^{t}, \quad f_{j}^{t}=\int_{t}^{\beta} R_{j, n-1}(t, u) B_{n} \ell^{u} d u \quad \text { for } j<n .
$$

We claim that these function satisfy (2.5). This is obvious for $k=n$. If $k<n$, then, by (2.6),

$$
\sum_{j=k}^{n} R_{k j}(r, t) f_{j}^{t}=R_{k n}(r, t) f_{n}^{t}+\sum_{j=k}^{n-1} \int_{t}^{\beta} R_{k j}(r, t) R_{j, n-1}(t, u) B_{n} \ell^{u} d u .
$$

By (1.8), the first term in the right-hand side of (2.7) is equal to

$$
\int_{r}^{t} R_{k, n-1}(r, s) B_{n} T_{n}(s, t) f_{n}^{t} d s=\int_{r}^{t} R_{k, n-1}(r, u) B_{n} \ell^{u} d u .
$$

By (1.14), the second term is equal to

$$
\int_{t}^{\beta} R_{k, n-1}(r, u) B_{n} \ell^{u} d u
$$




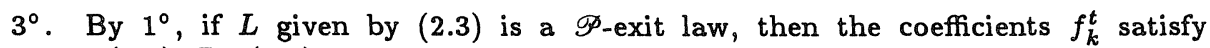
conditions (2.5). By (1.8), this implies

$$
\begin{aligned}
f_{n}^{r} & =T_{n}(r, t) f_{n}^{t}, \\
f_{k}^{r} & =T_{k}(r, t) f_{k}^{t}+\sum_{j=k+1}^{n} \int_{r}^{t} R_{k, j-1}(r, s) B_{j} T_{j}(s, t) f_{j}^{t} d s \quad \text { for } k<n .
\end{aligned}
$$

We conclude from this formula that $T_{k}(r, t) f_{k}^{t} \leqslant f_{k}^{r}$ for all $k$ and all $r<t<\beta$. Therefore $T_{k}(r, t) f_{k}^{t}$ is monotone decreasing in $t$, a limit $\ell_{k}^{r}=\lim _{t \rightarrow \beta} T_{k}(r, t) f_{k}^{t}$ exists and it does not exceed $T_{k}(r, t) f_{k}^{t}$ for all $k$.

By passing to the limit in formula $T_{k}(r, s) T_{k}(s, t) f_{k}^{t}=T_{k}(r, t) f_{k}^{t}$, we prove that $\ell_{k} \in \mathscr{L}_{\beta}\left(p_{k}\right)$. (The dominated convergence theorem is applicable since $T_{k}(s, t) f_{k}^{t} \leqslant f_{k}^{s}$ and $T_{k}(r, s) f_{k}^{s} \leqslant f_{k}^{r}<\infty$.)

Since $\ell_{j}^{s} \leqslant T_{j}(s, t) f_{j}^{t}$, formula (2.10) implies

$$
\begin{aligned}
& \int_{r}^{t} R_{k, j-1}(r, s) B_{j} \ell_{j}^{s} d s \leqslant \int_{r}^{t} R_{k, j-1}(r, s) B_{j} T_{j}(s, t) f_{j}^{t} d s \leqslant f_{k}^{r} \\
& \quad \text { for all } r<t<\beta \text { and all } k<j \leqslant n .
\end{aligned}
$$

Therefore,

$$
\int_{r}^{\beta} R_{k, j-1} B_{j} \ell_{j}^{s} d s \leqslant f_{k}^{r}<\infty .
$$

By passing to the limit in (2.10), we get

$$
f_{n}^{r}=\ell_{n}^{r}, \quad f_{k}^{r}=\ell_{k}^{r}+\sum_{j=k+1}^{n} \int_{r}^{\beta} R_{k, j-1}(r, s) B_{j} \ell_{j}^{s} d s \quad \text { for } k<n .
$$

By (2.3), (2.10) and (2.13),

$$
L^{t}(\mu)=\sum_{k=1}^{n}\left\langle f_{k}^{t}, \mu^{k}\right\rangle=\sum_{k=1}^{n}\left\langle\ell_{k}^{t}, \mu^{k}\right\rangle+\sum_{1 \leqslant k<j \leqslant n} \int_{t}^{\beta}\left\langle R_{k, j-1}(t, s) B_{j} \ell_{j}^{s}, \mu^{k}\right\rangle d s
$$

which implies (2.2). Theorem 2.1 is proved.

2.2. Examples. Fix $\beta<\infty$. To every function $f \in S$ there corresponds $\ell_{\beta, f}$ given by the formula

$$
\ell_{\beta, f}^{t}(x)=\int \prod_{1}^{n} p\left(t, x_{i} ; \beta, d y_{i}\right) f\left(y_{1}, \ldots, y_{n}\right)
$$

We say that $m$ is a reference measure for $p$ if the measure $p(r, x ; t, \cdot)$ is absolutely continuous with respect to $m$ for all $r<t$ and for all $x \in E$. By [4], the density function $\rho$ can be chosen to satisfy the equation

$$
\int \rho(r, x ; s, y) m(d y) \rho(s, y ; t, z)=\rho(r, x ; t, z)
$$

for all $r<s<t$ and for all $x, z \in E$. To every symmetric measure $\eta$ on $\left(E^{n}, \mathscr{B}^{n}\right)$ there corresponds $\ell_{\beta, \eta} \in \mathscr{L}_{\beta}\left(p_{n}\right)$ defined by the formula

$$
\ell_{\beta, \eta}^{t}(x)=\int \prod_{1}^{n} \rho\left(t, x_{i} ; \beta, y_{i}\right) \eta\left(d y_{1}, \ldots, d y_{n}\right) .
$$

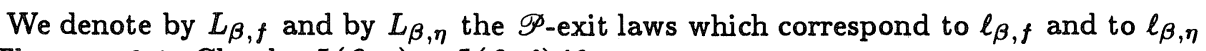
by Theorem 2.1. Clearly, $L(\beta, \eta)=L(\beta, f)$ if

$$
\eta\left(d y_{1}, \ldots, d y_{n}\right)=f\left(y_{1}, \ldots, y_{n}\right) m\left(d y_{1}\right) \cdots m\left(d y_{n}\right) \text {. }
$$




\section{Excessive functions}

Let $T(t)$ be the semigroup corresponding to a stationary transition function $p$. We say that a function $h \in p \mathscr{B}$ is $p$-excessive and we write $h \in \mathscr{H}(p)$ if $h(x)<\infty$ for some $x$, $T(t) h \leqslant h$ for all $t$ and $T(t) h \rightarrow h$ as $t \rightarrow 0$. A function $h \in \mathscr{H}(p)$ is called purely excessive if $T(t) h \rightarrow 0$ as $t \rightarrow \infty$ and it is called invariant if $T(t) h=h$ for all $t$. We denote the set of all purely excessive functions by $\mathscr{H}_{\text {pur }}(p)$ and the set of all invariant functions by $\mathscr{H}_{\text {inv }}(p)$.

Every $h \in \mathscr{H}(p)$ has a unique representation $h=h_{1}+h_{2}$, where $h_{1} \in \mathscr{H}_{\text {inv }}(p)$ and $h_{2} \in \mathscr{H}_{\text {pur }}(p)$.

Note that $\mathscr{H}_{\text {inv }}(p)$ coincides with the class of $t$-independent $p$-exit laws at $\infty$.

To every $\ell \in \mathscr{L}_{0}(p)$ there corresponds a function

$$
h=\int_{-\infty}^{0} \ell^{t} d t
$$

which is purely excessive unless it is equal to $\infty$ identically. The set of all purely excessive functions $h$ of the form (3.1) will be denoted by $\mathscr{H}_{\text {pur }}^{0}(p)$.

If there exists a reference measure for $p$, then, by [1] (Section 7.6), $\mathscr{H}_{\text {pur }}(p)=\mathscr{H}_{\text {pur }}^{0}(p)$.

Theorem 3.1. To every $h \in \mathscr{H}_{\mathrm{pur}}^{0}\left(p_{n}\right)$ there corresponds $H_{h} \in \mathscr{H}_{\mathrm{pur}}(\mathscr{P})$ defined by the formula

$$
H_{h}(\mu)=\left\langle h, \mu^{n}\right\rangle+\sum_{j=1}^{n-1}\left\langle G^{j} B_{j+1} G^{j+1} \cdots B_{n} h, \mu^{j}\right\rangle,
$$

where $G^{j}$ is given by (1.15) with $\lambda=0$.

$\mathrm{P} \mathrm{r} \circ$ of. Let $\ell$ correspond to $h$ by (3.1) and let $L \in \mathscr{L}_{0}(\mathscr{P})$ correspond to $\ell$ by Theorem 2.1. Then

$$
H_{h}(\mu)=\int_{-\infty}^{0} L^{t}(\mu) d t
$$

belongs to $\mathscr{H}_{\text {pur }}(p)$. Formula (3.2) follows from (3.3), (2.1), (1.9), (1.17).

\section{REFERENCES}

1. Dynkin E. B. Minimal excessive measures and functions. - Trans. Amer. Math. Soc., 1980 , v. 258 , p. $217-244$.

2. Dynkin E. B. Superprocesses and their linear additive functionals. - Trans. Amer. Math. Soc., 1989, v. 314, p. 255-282.

3. Dynkin E. B. An Introduction to Branching Measure-Valued Processes. Providence, RI: Amer. Math. Soc., 1994, 134 p.

4. Дынкия E. Б., Кузяецов С. E. Определяюшие функции марковских процессов и соответствуюшие сопряженные регулярные классы. - Докл. АН СССР, 1974, T. 214, № 1 , c. 25-28.

5. Roelly-Coppuletta $S$. A criterion of convergence of measure-valued processes: application to measure branching processes. - Stochastics, 1986, v. 17, p. 43-65. 